Voix et Images

voixetimages

\title{
Une « relève " culturelle et nationale
}

\section{Robert Dion}

Volume 29, numéro 1 (85), automne 2003

\section{Claire Martin}

URI : https://id.erudit.org/iderudit/007548ar

DOI : https://doi.org/10.7202/007548ar

Aller au sommaire du numéro

\section{Éditeur(s)}

Université du Québec à Montréal

\section{ISSN}

0318-9201 (imprimé)

1705-933X (numérique)

Découvrir la revue

Citer ce compte rendu

Dion, R. (2003). Compte rendu de [Une « relève » culturelle et nationale]. Voix et Images, 29(1), 155-158. https://doi.org/10.7202/007548ar d'utilisation que vous pouvez consulter en ligne.

https://apropos.erudit.org/fr/usagers/politique-dutilisation/ 


\section{R E C H E R C H E}

Une «relève»culturelle et nationale

$++$

ROBERT DION

Université du Québec à Montréal

Dans l'imaginaire populaire, jeunesse et poésie vont, semble-t-il, de pair : les émois et épanchements poétiques auraient ainsi partie liée avec les difficultés de l'adolescence, et l'âge adulte serait précisément marqué par un «dépassement» du lyrisme, pour parler comme Milan Kundera (ou comme son interprète québécois François Ricard). Certes, les exemples sont légion de jeunes gens dont la ferveur poétique n'aura duré que l'espace de la périlleuse traversée de l'âge ingrat. Mais il y a, d'une part, de nombreux exemples de réussites éclatantes imputables à de très jeunes poètes et, d'autre part, un nombre non moins important de cas où la passion de la poésie a perduré bien au-delà du cap de la vingtaine et de ses tourments. C'est en l'occurrence ce qui s'est produit chez certains membres du petit groupe à l'origine de la fondation, en 1953, des Éditions de l'Hexagone et dont Christine Tellier, dans Jeunesse et poésie. De l'Ordre de Bon Temps aux Éditions de $l^{\prime}$ Hexagone, retrace la trajectoire et les premières réalisations ${ }^{1}$.

Une telle «exploration de la préhistoire et des premières années de l'Hexagone» (12) ne s'effectue pas en territoire absolument vierge. Tellier prend d'ailleurs soin de rappeler ce qu'elle doit aux travaux précurseurs de Gilles Marcotte et de Jean-Louis Major. Mais elle va plus loin, d'abord en exploitant de manière systématique les fonds d'archives (de l'Hexagone, de l'Ordre de Bon Temps [OBT], ainsi que de Gaston Miron, Ambroise Lafortune, Roger Varin, entre autres), ensuite en ayant recours à un corpus considérable d'interviews réalisées entre 1997 et 2002. Ce faisant, elle parvient à montrer comment se constitue le réseau de l'OBT - mouvement de jeunesse actif entre 1946 et 1956, voué à la promotion de loisirs culturels populaires fondés sur la participation et inspirés des traditions canadiennes-françaises - et comment, de ce premier réseau, se dégage peu à peu l'équipe de six personnes qui se soudera autour du projet d'édition du recueil des poèmes de Gaston Miron et Olivier Marchand, Deux sangs. Parallèlement, l'auteure indique par quelles ramifications l'OBT s'insère dans tout un ensemble d'organismes - Jeunesse étudiante catholique (JEC), Jeunesse ouvrière catholique (JOC), clans de routiers, et ainsi de suite qui, de façon convergente mais non sans quelques tiraillements parfois, travaillèrent, sur une base plus concrète qu'idéologique, au renouveau culturel québécois dans le cadre de ce

$+++$

1 Christine Tellier, Jeunesse et poésie. De l'Ordre de Bon Temps aux Éditions de l'Hexagone, Montrêal, Fides, coll. «Nouvelles Études québécoises", 2003, 334 p. 
qui apparaît comme une sorte de pré-«Révolution tranquille». Elle fait aussi voir à quel point l'Ordre fut une pépinière de talents pour diverses institutions culturelles des années 1950 et 1960 - radio et télévision de Radio-Canada, Office national du film, théâtres et, bien sûr, maisons d'édition ${ }^{2}$.

Lorsqu'ils décident de lancer une souscription pour publier les poèmes de deux des leurs, les jeunes Gilles Carle, Jean-Claude Rinfret, Gaston Miron, Olivier Marchand, Mathilde Ganzini et Louis Portugais, les six «côtés» du futur Hexagone, n'ont pas le sentiment, tant s'en faut, de participer à ce qui deviendra une percée majeure de l'édition et de la littérature québécoises. Leur position institutionnelle leur interdit absolument une telle ambition. Ils se situent en effet en marge, sinon en-dehors, du milieu littéraire, et cette situation «exotopique» se traduit dans leurs choix esthétiques : souhaitant pratiquer une poésie qui «s'inscrive dans la vie de la communauté» (26), voulant rompre avec la problématique de la solitude et de l'échec, valorisant une prise de parole collective (d'où une première publication «à quatre mains»), ils se dissocient de l'esthétique des grands aînés - Alain Grandbois, Saint-Denys Garneau, Anne Hébert - , de ces «monuments» isolés pratiquant une poésie jugée élitiste, au demeurant alignée sur l'esthétique parisienne $(198)^{3}$. Tellier met clairement en relief l'ancrage de ces jeunes de l'OBT et du Clan Saint-Jacques dans la vie communautaire et l'action sociale plutôt que dans les cercles artistiques ou bourgeois ${ }^{4}$; elle circonscrit avec précision le profil d'une génération qui se reconnaît dans un certain catholicisme progressiste (d'où l'importance du père Ambroise Lafortune au carrefour des divers mouvements de jeunesse), qui aspire à une vie saine et active, qui a le sens de l'initiative, sait faire beaucoup avec peu et estime que le relèvement culturel de la nation ne passe ni par les discours des idéologues ni par les structures rigides (celles de l'Église, par exemple), mais par l'effort d'un groupe partageant les mêmes valeurs.

Dans ce bon travail d'histoire littéraire, la chercheuse souligne également tout ce que l'aventure de l'Hexagone doit à l'amitié, à des convergences inopinées, aux tâtonnements d'un apprentissage de l'écriture et de l'édition largement autodidacte, à des entreprises menées en amateur (entre autres la publication de La Galette et du Godillot, organes de l'OBT et du Clan Saint-Jacques respectivement). Elle nous fait assister de près aux balbutiements de l'une des premières institutions éditoriales québécoises modernes, à ses débuts non seulement artisanaux mais très modestes, malgré le succès critique et commercial de Deux sangs (grâce à la souscription et aux ventes à l'unité, le recueil fait ses frais, rapportant même la faramineuse somme de... 11,57\$!): on a tendance à l'oublier aujourd'hui, mais l'Hexagone ne devait au départ exister que pour cette seule publication, et Miron, s'il avait jusque-là dirigé les bulletins des mouvements de jeunesse auxquels il adhérait, ne pouvait alors savoir que l'édition serait l'une de ses vocations. D'ailleurs l'équipe initiale se dispersera bientôt, chacun suivant ses propres penchants et entamant une carrière ayant peu à voir avec l'édition de poésie. Mais entre-temps l'Hexagone aura vu le jour et d'autres personnes, autour de Miron, pousseront l'aventure un peu plus loin.

2 En annexe, l'auteure publie un long texte de 1993 signé par Guy Messier (un ex-membre de l'OBT), qui relève systématiquement l'apport des sympathisants de l'Ordre à divers secteurs de la culture québécoise au fil des décennies suivantes. 3 «Je rêve d'une poésie populaire de grand art", écrit Miron dans une lettre à Andrée Maillet-Hobden; citế par Tellier, p. 189. 4 Plusieurs viennent de l'extêrieur de Montréal et sont issus de milieux populaires. 
Si les jeunes membres de l'OBT réagissent à la conjoncture culturelle de l'après-guerre par l'action concrète en essayant de se tenir à distance des pouvoirs, des structures contraignantes et des idéologies trop tonitruantes, ce n'est certes pas le cas du très doctrinaire chanoine Groulx, qui poursuit l'œuvre d'historien, de conférencier et de propagandiste entamée dans les années 1910. D'une certaine façon - et c'est ce que le livre de Gérard Bouchard, Les deux chanoines. Contradiction et ambivalence dans la pensée de Lionel Groulx ${ }^{5}$, se trouve à illustrer indirectement - , l'action de Groulx, amateur de philippiques prononcées ex cathedra, grand discoureur et intarissable idéologue, se situe aux antipodes du type d'intervention préconisé par les mouvements de jeunesse.

Dans l'ouvrage très documenté qu'il consacre à une question on ne peut plus délicate, Bouchard choisit de se placer au-dessus des polémiques récentes concernant le chanoine. À la suite d'une lecture exhaustive de ses publications - tâche titanesque s'il en est, et combien méritoire! - , il s'attache à produire une vision globale de sa pensée qui puisse déboucher sur un bilan dépassionné de son action. Il y a pourtant quelque chose, dans ce livre, d'un "pour en finir avec Lionel Groulx» : même si le ton reste serein et l'argumentation patiente, on décèle çà et là un certain agacement vis-à-vis de l'attention démesurée dont a fait l'objet une œuvre que Bouchard, au bout du compte, juge dépassée et assez peu pertinente pour le temps présent ${ }^{6}$. C'est dans cet état d'esprit qu'il entreprend de décrire «un univers intellectuel foncièrement incohérent, imprégné de contradictions non surmontées » (10). La thèse de Bouchard est en effet que Groulx, en raison de sa formation, de ses idéaux, de ses fidélités et du contexte dans lequel il a vécu, a été incapable de résoudre les contradictions de sa pensée. Bien sûr, aucune pensée n'est monolithique ni exempte de tensions; toutefois, chez le chanoine, ces incompatibilités ne seraient pas accessoires mais structurelles. Elles produiraient, selon Bouchard, un type de pensée équivoque, c'est-à-dire une pensée qui, pour «harnacher» (le terme est de lui) les contradictions du réel, propose «un montage hétéroclite, un simple alignement d'énoncés contraires, sans mise en tension, sans effet de levier» (23). Alors que les pensées organique et radicale sont à même de produire des mythes compensatoires rémunérant des contradictions insurmontables par la raison (par exemple, aux États-Unis «l'idéologie égalitariste [...] est contredite par l'ampleur et la permanence des inégalités, mais rachetée par le mythe de la mobilité sociale et de l'achievement, au cœur de l'American dream » - 23), la pensée équivoque n'arrive pas à élaborer des représentations totalisantes qui s'accordent avec les données et les urgences de l'actuel. Ainsi, Groulx aurait formulé des mythes inefficaces, anachroniques, tels ceux d'une Nouvelle-France idyllique, de la race héroïque de nos pères, de la mission du petit peuple canadien-français. Dans ce caractère inopérant de la production mythique du chanoine résiderait du reste l'un des motifs de ce que Bouchard appelle son «échec»: car «à une époque d'industrialisation et d'urbanisation accélérée, quel parti, quelle élite ou corporation, quelle classe sociale aurait trouvé un intérêt immédiat à se mettre à la remorque de ce genre de discours?» (242)

Je n'entends pas ici prendre position dans le débat autour de la figure de Groulx: la question est trop vaste pour une rapide chronique. J'insiste plutôt sur le projet même de Bouchard et sur sa réalisation. Celui-ci, donc, a voulu se démarquer des nombreuses lectures parcellaires et partiales de Groulx effectuées récemment. Son parti pris de

$+++$

5 Gêrard Bouchard, Les deux chanoines. Contradiction et ambivalence dans la pensée de Lionel Groulx, Montréal, Boréal, 2003, 313 p. 6 À ce sujet, voir notamment la conclusion du volume. 
totalité le conduit à discerner chez le chanoine, derrière certaines cohérences «localisées», "sectorielles», une pensée foncièrement contradictoire et, partant, inutilisable pour ses successeurs: une pensée qui affirme, avec la même autorité et parfois la même violence, une chose et son contraire. Par exemple, l'auteur de L'appel de la race est indépendantiste et en même temps (non successivement) fédéraliste, démocrate et fasciste, conservateur et moderne, libéral et réactionnaire, philosémite et antisémite, et ainsi de suite ${ }^{7}$. Afin de le prouver, Bouchard reprend chacune de ces oppositions, qu'il éclaire par de nombreuses citations tirées de l'œuvre. Pour convaincant et nécessaire qu'il apparaisse, le procédé n'en est pas moins lassant par ce qu'il induit de répétitions (certaines citations, notamment, reviennent à de multiples reprises), de symétries (chaque chapitre est construit de façon identique), d'inventaires un peu fastidieux. Alors que le premier chapitre, qui décrit la démarche, et les derniers (XIV, XV et XVI spécialement), qui exposent les conclusions, sont fort stimulants, les sections médianes, quoique agréablement rédigées, rappellent parfois davantage les fiches de lecture du travail préparatoire que le discours articulé de l'historien. Sans doute peut-on imputer cette relative monotonie de l'analyse de discours à l'absence d'arrière-plan théorique autre que le concept de mythe fédérateur et compensatoire. Du coup, l'analyse, trop descriptive, ne décolle guère du corpus et n'éclaire pas toujours suffisamment certaines questions délicates comme le fascisme du chanoine (simplement renvoyé à l'idéologie corporatiste, à la mystique du chef et de la regénération) ou son racisme-antisémitisme (évalué à l'aune de la distinction - fragile - entre antisémitisme et antijudaïsme).

Ces quelques réserves mises à part, le bilan que tire Bouchard de l'œuvre de Groulx apparaît tout à fait pertinent. Plus particulièrement dans les derniers chapitres, le chercheur va au-delà de la description et se risque à expliquer les contradictions fondamentales du chanoine. Bouchard relève ainsi l'opposition entre Groulx le visionnaire et Groulx le scientifique (195); il montre comment des visions incompatibles de la nation s'affrontent chez lui; il observe les ambivalences de sa conception de l'histoire, tantôt perçue comme résultante de l'action libre des hommes, tantôt comme l'expression de la Providence. Groulx aurait en outre échoué à mobiliser durablement les élites faute d'un programme un tant soit peu consistant; il aurait été entraîné par son lyrisme à sacrifier la cohérence intellectuelle à l'humeur du moment; il aurait été incapable, en vertu de sa position institutionnelle, «de penser ni le statu quo ni le changement, ni la tradition ni la modernité, ni la continuité (francité) ni la rupture (américanité)» (237). Quant aux mythes proposés par Groulx, ils seraient, on l'a vu, trop monosémiques et trop décalés par rapport à l'actualité. Enfin, au lieu d'être radicale comme on le croit souvent, sa pensée serait restée équivoque à cause de l'inaptitude du chanoine à mener jusqu'à son terme la réflexion sur son nationalisme, son fascisme, son libéralisme, son humanisme (245). Bref, c'est à une évaluation sévère de la pensée de Groulx que procède Bouchard dans Les deux chanoines, et il semble à tout prendre que, même si l'auteur de Notre maître, le passé, "homme morcelé, dressé contre lui-même» (248), reflète avec assez de justesse une société alors déchirée et désemparée, il n'y ait plus grand-chose à sauver de son œuvre: Groulx ne constitue plus désormais qu'un objet du discours historique, certainement pas l'une de ses inspirations.

$+++$

7 Je reprends ici en partie certains intitulês des chapitres du livre de Bouchard. 